\title{
Uso de herramientas digitales en un estudio de tendencias de movilidad en bicicleta en Ciudad del Conocimiento de la UAEH
}

\author{
Use of digital tools in a study of bicycle mobility trends in UAEH's Knowledge City
}

\author{
Yoan Beltrán-Martínez ${ }^{\mathrm{a}, *}$ (iD), Jairo Lozano-Hernández ${ }^{\mathrm{b}}$ (D), Catherine Ramírez-Islas ${ }^{\mathrm{c}}$ (i) \\ a bc Área Académica de Ingeniería y Arquitectura, Universidad Autónoma del Estado de Hidalgo, 42184, Pachuca, Hidalgo, México.
}

\begin{abstract}
Resumen
La movilidad urbana se constituye es un tema relevante para el diseño de ciudades, con el objetivo de asegurar la plena accesibilidad a todos sus habitantes. Es importante incorporar nuevas herramientas digitales para su estudio. En este artículo se aborda el uso de tecnología digital como herramienta para medir la tendencia del uso de la bicicleta en entornos académicos, tomando como caso de estudio a la Ciudad del Conocimiento de la Universidad Autónoma del Estado de Hidalgo, México. Se revisan tendencias y preferencias de usuarios, las posibilidades que existen para la implementación de una estrategia de movilidad en bicicleta, así como recomendaciones hacia el interior de las instituciones de educación superior (IES) e involucrados en la conformación de la ciudad.
\end{abstract}

\section{Palabras Clave:}

Movilidad, bicicleta, diseño, tecnología digital.

\begin{abstract}
Urban mobility is a relevant issue for city design in order to ensure full accessibility to all its inhabitants. It is important to incorporate new digital tools for your study. This article focuses on the usage of digital technology as a genesis to measure the bicycle usage in university context, taking as a study case Ciudad del Conocimiento, campus UAEH (Universidad Autónoma del Estado de Hidalgo), Mineral de la Reforma, Hidalgo. Tendencies and preferences of users are reviewed, possibilities for the application of bicycle mobility strategy, although, as the guidance inside education institutions of higher education (IES) muddled inside city conformation.
\end{abstract}

Keywords:

Urban mobility, bicycle, design, digital technology.

\section{Introducción}

\subsection{La movilidad urbana}

La forma en las que las personas se mueven por las ciudades ha cambiado de forma importante en los últimos años. No solo por el medio en la que las personas se desplazan, sino en los motivos y la forma en la que lo llevan a cabo. (Herce, 2009). No es de extrañar que bajo una perspectiva de modernidad el desplazamiento se vincule directamente con el transporte. Sin embargo, no debemos olvidar que los primeros desplazamientos se hicieron a pie, y en algunas ocasiones llevando consigo cargas. A inicios del siglo XX, en el periodo comprendido entre 1928-1960, los esfuerzos de organismos por un mejor entorno urbano se vieron plasmados en el Congreso Internacional de Arquitectura Moderna (CIAM), una serie de conferencias en donde se involucraban temas de urbanidad, espacio público y formas de desplazamiento, entre varios temas (Mumford, 2002). Dentro de muchos de los resultados, la llamada Carta de Atenas fungió como un impulso para las naciones en vías de repensar la forma en la que las ciudades se diseñaban.

\footnotetext{
*Autor para la correspondencia: yoans@uaeh.edu.mx Islas)

Correo electrónico: yoans@uaeh.edu.mx (Yoan Saidt Beltrán Martínez), jairo_lozano@uaeh.edu.mx (Jairo Armando Lozano Hernández), a322507@uaeh.edu.mx (Catherine Ramírez 
Sin embargo, este discurso seguía regido por un ánimo de modernidad que veía en la industrialización y la tecnología, el camino para el desarrollo. El transporte sería uno de los elementos que más notorios en avances tecnológicos en este proceso de modernización, en donde se buscaba que los desplazamientos fueran mayores y con una gran velocidad.

Moverse en la ciudad se ha conceptualizado como desplazarse en un medio de transporte. Alejada del concepto de transporte, la movilidad urbana se define como el deseo de desplazamiento de un lugar a otro para satisfacer necesidades de trabajo, estudio, recreación, u otro que el ser humano requiera.

Así como la preocupación de la movilidad y transporte han sido fundamentales para la gestión de las ciudades, el tema del medio ambiente ha cobrado fuerza debido a un sin número de acontecimientos. Sin embargo esta relación histórica entre medio ambiente y desarrollo se ha manifestado como acciones que llevan a la dominación y explotación de los recursos, los cuales están asociados con una visión eminentemente utilitarista (Grant, 2006).

\subsection{La bicicleta como elemento estratégico de movilidad urbana sustentable en la Zona Metropolitana de Pachuca}

Si la movilidad urbana se analiza como desplazamientos, y estos desplazamientos inevitablemente consumen recursos, es preciso retomar la conceptualización de movilidad urbana sustentable al incluirla como una serie de acciones encaminadas a satisfacer las necesidades de desplazamiento sin un consumo excesivo de los recursos ambientales, materiales, sociales, para su aprovechamiento actual, pero con énfasis en considerar que los desplazamientos, sus políticas de movilidad urbana, no desemboquen en un distanciamiento entre las clases dirigentes y el resto de la población (Lange, 2011, p. 97).

En este sentido, la comprensión de la movilidad urbana a partir de un concepto de sustentabilidad se vuelve determinante en ciudades latinoamericanas (Lizárraga, 2006), no solo porque la repercusión en temas ambientales resulta preocupante, sino porque esto deriva en situaciones sociales y económicas: enfermedades producidas por el sedentarismo y la contaminación, así como los altos costos que involucra la gestión del espacio publico.

Los modos de transporte en la Zona Metropolitana de Pachuca (ZMP) han respondido a un sistema estructurado a partir del modelo de demanda. Así, la inclusión de estos sistemas ha respondido a las necesidades de los desplazamientos de las personas, principalmente por motivos de viaje al trabajo y motivos de viaje a la escuela.

Es entonces como la forma de reconstruir la educación cívica y ambiental es mediante la propuesta de modificar la forma en la que vemos a la denominada "cultura del automóvil", que no solo pide la conciencia sobre la racionalización de éste medio de transporte, sino el fomento de políticas aplicadas a la movilidad urbana (Cárdenas, 2015)

$\mathrm{Si}$ a lo anterior añadimos variables como el lugar de residencia, se puede comprender que el sistema de movilidad requiere de una estructura cada vez mejor articulada. De acuerdo a los viajes por Municipio de la ZMP (Cal y Mayor \& Asociados, 2012), 179,198 viajes son realizados en Mineral de la Reforma, en cambio, en Pachuca se realizan un total de
681,383 viajes; resultando un total de 1,070,362 viajes dentro de los municipios de Pachuca, San Agustín Tlaxiaca, Mineral del Monte, Epazoyucan, Villa de Tezontepec, Mineral de la Reforma y Zempoala, que comprenden a la Zona Metropolitana de Pachuca, correspondiente a 2.1 viajes por persona. Este número nos indica que las personas realizan más de dos viajes al día. La ciudad de Pachuca se acerca más a los tres viajes por día, lo que podría explicarse debido a los transbordos y distintos factores que influyen en la movilidad urbana de las personas.

Tabla 1: Cantidad de viajes en la Zona Metropolitana de Pachuca

\begin{tabular}{cccc}
\hline Municipio & $\begin{array}{c}\text { No. de } \\
\text { viajes }\end{array}$ & Población & $\begin{array}{c}\text { Viajes/ } \\
\text { Persona }\end{array}$ \\
\hline Pachuca de Soto & 681,383 & 269,480 & 2.5 \\
San Agustín Tlaxiaca & 69,861 & 32,290 & 2.2. \\
Mineral del Monte & 27,320 & 13,877 & 2.0 \\
Epazoyucan & 27,147 & 13,914 & 2.0 \\
Villa de Tezontepec & 20,151 & 11,735 & 1.7 \\
Mineral de la Reforma & 179,198 & 130,980 & 1.4 \\
Zempoala & 65,302 & 39,509 & 1.7 \\
\hline
\end{tabular}

Fuente: Cal y Mayor \& Asociados. (2012). Proyectos y estudios del tren suburbano en la región sur del Estado de Hidalgo y zonas metropolitanas del Estado de México y Ciudad de México. Secretaria de Obras Públicas y Ordenamiento Territorial del Gobierno del Estado de Hidalgo

En las mejores prácticas del urbanismo sustentable, se argumenta que más allá de conceptualizar un rol o impacto en términos de política de transporte, es la creación, diseminación y aplicación de estrategias en materia de diseño urbano, las que promueven un desarrollo eficaz de las ciudades (Lezama \& Domínguez, 2006, p. 153-176).

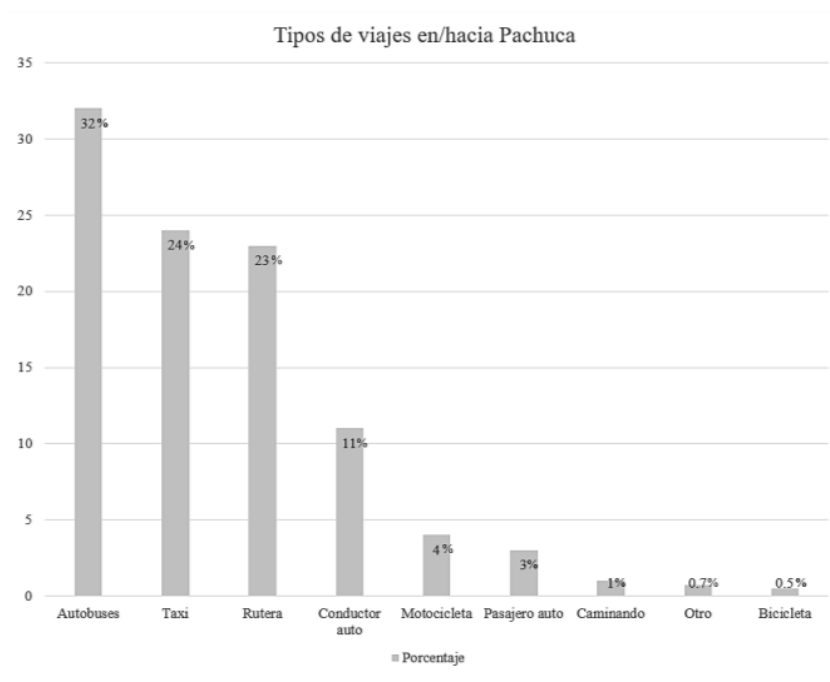

Grafico 1: Viajes dentro del Municipio de Pachuca

Fuente: Cal y Mayor \& Asociados. (2012). Proyectos y estudios del tren suburbano en la región sur del Estado de Hidalgo y zonas metropolitanas del Estado de México y Ciudad de México. Secretaria de Obras Públicas y Ordenamiento Territorial del Gobierno del Estado de Hidalgo

De esta manera, el uso de la bicicleta como medio de transporte o modo de movilidad, se ha visto caracterizado por un impulso en las ciudades latinoamericanas. Desde la 
perspectiva de la movilidad urbana sustentable, la bicicleta se ha posicionado como una opción a algunos problemas en las ciudades. Sin embargo, se cae fácilmente en considerar a la bicicleta como un modo lúdico, dejando de lado su potencial como modo de transporte.

\subsection{Movilidad urbana en entornos universitarios}

Los equipamientos urbanos que contienen las ciudades (hospitales, mercados, escuelas, etc.) son parte importante de la estructura urbana, no solo como elementos que conforman el paisaje, sino como elementos que funjan como "atractores" y determinantes de las distintas dinámicas de una ciudad. Un parque, una plaza pública o una escuela modifican notablemente la forma de habitar ciertas zonas de la ciudad que las albergan.

En el caso de las universidades mexicanas, al ser instituciones que atraen a un gran sector de las ciudades que las albergan, e incluso fuera de ellas, resulta interesante saber los modos de desplazamiento que hay hacia ellas, así como las tendencias a moverse de formas alternativas.

Casos como el de el programa Campus sustentable, de la Universidad de Sao Paulo, Brasil (Vilela, Grimoni, \& Burani, 2010), UNIBICI, de la universidad de Madrid (Monzón, La Paix, \& Rondinella, 2008) o el de ECOPUMA en la UNAM, México (Imaz, Ayala, Gutiérrez, \& González, 2015) han sido claros ejemplos de que la los desplazamientos hacia y en entornos universitarios son temas de la movilidad urbana que deben atenderse con profundo interés, no solo para las instituciones, sino para la estructura y crecimiento de las ciudades.

En este sentido, la importancia de estudiar el caso de los desplazamientos y tendencias de los modos de movilidad en la Universidad Autónoma del Estado de Hidalgo radica en la portación de datos para futuras mejoras en el funcionamiento del esquema de movilidad hacia dicho equipamiento y al interior del miso, con una visión de integración al tejido de la ciudad en materia de diseño urbano, tomando en cuenta estrategias y herramientas para la movilidad en bicicleta, sin dejar de lado el tema de movilidad peatonal.

\subsection{Tecnología digital como herramienta en los estudios urbanos}

Los avances en tecnología han permitido que distintas disciplinas amplíen los horizontes de trabajo e investigación. En el caso de las ciudades, la tecnología ha permitido generar nuevas herramientas de análisis, diseño y producción de las mismas. No es extraño encontrarnos con investigaciones y aproximaciones metodológicas que contemplan la relación entre tecnología y ciudad. Desde ciudades tecnológicas (Downey \& McGuigan, 1999) (Castells \& Hall, 1994), ciudades inteligentes (Telefónica, 2011) (Townsend, 2013) (Campbell, 2013) o ciudades digitales (Ishida \& Isbister, 2000) (Mossberger, Tolbert, \& Franko, 2013), el uso de la tecnología digital ha acompañado a las distintas etapas de las ciudades en la revolución tecnológica en materia digital.

Sin embargo, por lo novedoso de los métodos y, en ocasiones, la complejidad de su uso, éstas tecnologías no han sido accesibles para todas las personas o instituciones involucradas en el diseño de las ciudades. Los costos de licencias, el uso de herramientas especializadas, así como la curva de aprendizaje, pueden ser un factor determinante para que los estudios de la ciudad sean conducidos por instancias especializadas o por entidades gubernamentales, dejando de lado a las organizaciones de la sociedad civil, a cierto sector de la academia que no cuenta con los recursos necesarios para los estudios, o a cualquier interesado en los temas urbanos a nivel de investigación.

En este sentido, iniciativas no comerciales o gubernamentales han surgido para cubrir el las necesidades de herramientas y datos generados a partir de elementos digitales. Conceptos como open source y crowdsourcing, que permiten que mediante una estrategia de colaboración, los datos y las herramientas digitales puedan estar al alcance de personas con recursos básicos. Lo anterior, dado que el uso de tecnologías digitales colaborativas, permiten abordar los problemas urbanos desde escalas mínimas hasta proyectos de gran envergadura.

\section{Metodología}

Para el estudio en cuestión, sobre movilidad hacia CC$\mathrm{UAEH}$, la referencia teórica y bibliográfica nos orientaron hacia ejercicios aplicados en Europa y América. Se decidió tomar como referencia el trabajo del Equipo UNIBICI (2008) de la Universidad de Madrid, principalmente por la disposición de información de la encuesta, así como los reportes detallados que se presentan para este caso, tomando como referencia fenómenos similares de desplazamiento.

De ésta forma, se estructura la metodología bajo el siguiente esquema:

- Revisión teórica sobre movilidad urbana, movilidad urbana sustentable, movilidad no motorizada y movilidad en entornos universitarios.

- Determinación de edificios que conforman a la CC-UAEH mediante Google Earth.

- Determinación de cuadrantes de ubicación mediante software de información geográfica QGIS.

- Diseño de la encuesta Google Forms.

- Diseño y campaña de difusión de la encuesta.

- Aplicación de la encuesta mediante envío online (correo electrónico), mensajería móvil (WhatsApp) y redes sociales oficiales de la universidad (Facebook)

- Análisis de los datos.

Diversas metodologías para estudios de movilidad se centran en recabar datos del usuario o potencial usuario mediante el uso de encuestas. El instrumento permite recabar información mediante una serie de preguntas que arrojan resultados a partir de los objetivos planteados en la investigación.

\section{El área de estudio}

\subsection{El entorno universitario}

Para el estudio se tomó en consideración a las instalaciones de la Ciudad del Conocimiento de la UAEH (CC-UAEH), 
antes conocida como Ciudad Universitaria, ubicada en el municipio de Mineral de la Reforma, Hidalgo. Dicho municipio es parte de la Zona metropolitana de Pachuca que, al censo de 2010, concentraba a 130,980 habitantes. Tan solo el área que abarca la CC-UAEH es de 304,434 $\mathrm{m}^{2}$ (UAEH, 2018), en ella concentra a una población aproximada de 7,500 usuarios, entre alumnos, profesores, personal administrativo y de servicios. Dicho centro de estudios se toma como lugar de atracción de distintos modos de desplazamiento, dadas las características y los distintos usos que tiene las instalaciones (educativos, deportivos, laborales, comerciales).

Los edificio que conforman la $\mathrm{CC}-\mathrm{UAEH}$ son:

- (CAI) Autoacceso

- AA Biología

- AA Ciencias de la tierra y materiales

- AA Ingeniería y arquitectura

- AA Química

- Biblioteca Central

- CEDAI

- CEDAI

- CEVIDE

- CITIS

- DUI

- Edificio A

- Edificio B

- Edificio C

- Edificio D

- Edificio F

- Edificio G

- Edificio $\mathrm{H}$

- Edificio I

- Eventos Especiales

- MF1

- MF2

- $\mathrm{MF3}$

- Odontología

- Pabellón Universitario

- Polideportivo

- Prepa UNO

- Química en Alimentos

- UCL

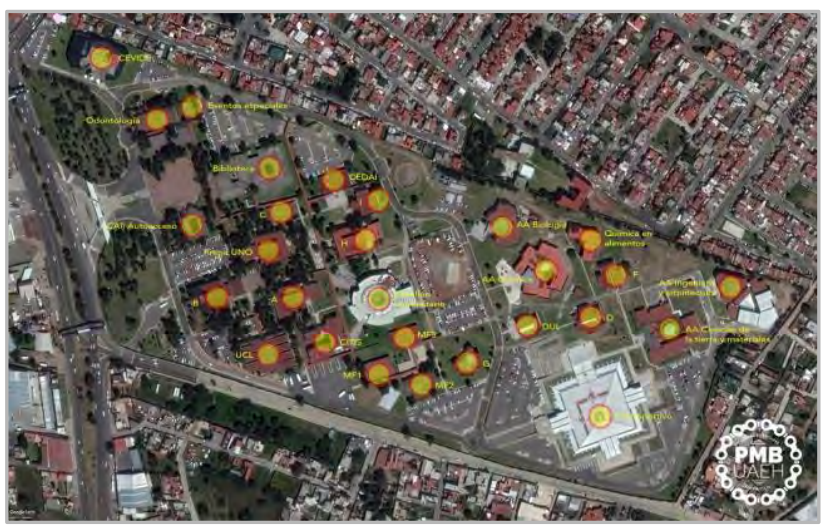

Figura 1: Mapa de ubicación de los edificios al interior de Ciudad del Conocimiento de la UAEH en el Municipio de Mineral de la Reforma, Hidalgo.

Fuente: Elaboración propia

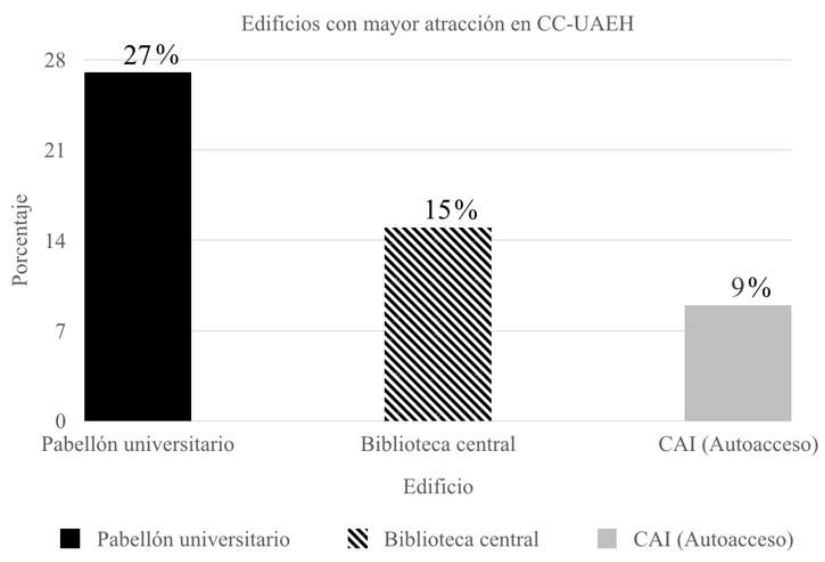

Gráfico 2: Edificio de CC-UAEH con mayor atracción de viajes Fuente: Elaboración propia

\subsection{Radio de servicio y determinación de cuadrantes de ubicación}

Por otro lado, para establecer el lugar de origen de los viajes hacia CC-UAEH, se tomó como lugares de atracción a las colonias cercanas a la Institución estableciendo cuadrantes de servicio para determinar la zona de precedencia. Tanto para los lugares de origen como de destino en CC-UAEH, se utilizó la herramienta Google Maps en su versión on line, obteniendo imágenes satelitales para su edición y posterior distribución en conjunto con la encuesta y como parte de la estrategia de difusión.

Dada la importancia de los dos municipios con mayor población, se optó por establecer tres grandes grupos de cuadrantes. En el primer grupo, se encuentran los cuadrantes correspondientes a Pachuca, en el segundo grupo de cuadrantes, se encuentran los correspondientes al Municipio de Mineral de la Reforma. Finalmente, se optó por establecer el lugar de origen a alguno de los otros cinco municipios restantes de la Zona Metropolitana de Pachuca, lo anterior dada la atracción que representa la UAEH como Institución de Educación Superior (IES)

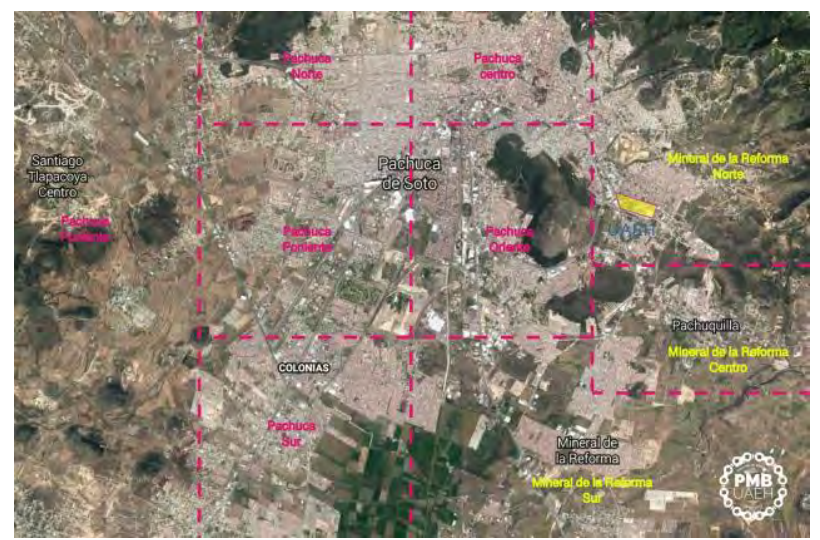

Figura 2: Mapa de ubicación de cuadrantes de origen de los viaje Fuente: Elaboración propia

\begin{tabular}{llcl}
\hline Municipio & Cuadrante & $\begin{array}{c}\text { Población } \\
\text { muestral }\end{array}$ & Porcentaje \\
\hline
\end{tabular}




\begin{tabular}{|c|c|c|c|}
\hline \multirow{4}{*}{$\begin{array}{l}\text { Pachuca de } \\
\text { Soto }\end{array}$} & Pachuca Centro & 90 & $8.6 \%$ \\
\hline & Pachuca Norte & 63 & $6.0 \%$ \\
\hline & Pachuca Oriente & 66 & $6.3 \%$ \\
\hline & Pachuca Poniente & 73 & $6.9 \%$ \\
\hline \multirow{4}{*}{$\begin{array}{l}\text { Mineral de } \\
\text { la Reforma }\end{array}$} & Pachuca Sur & 118 & $11.3 \%$ \\
\hline & $\begin{array}{l}\text { Mineral de la } \\
\text { Reforma Centro }\end{array}$ & 97 & $9.2 \%$ \\
\hline & $\begin{array}{l}\text { Mineral de la } \\
\text { Reforma Norte }\end{array}$ & 300 & $28.7 \%$ \\
\hline & $\begin{array}{l}\text { Mineral de la } \\
\text { Reforma Sur }\end{array}$ & 78 & $7.5 \%$ \\
\hline Epazoyucan & Epazoyucan & 5 & $0.47 \%$ \\
\hline $\begin{array}{l}\text { Mineral del } \\
\text { Monte }\end{array}$ & Mineral del Monte & 11 & $1.0 \%$ \\
\hline $\begin{array}{l}\text { San Agustín } \\
\text { Tlaxiaca }\end{array}$ & $\begin{array}{l}\text { San Agustín } \\
\text { Tlaxiaca }\end{array}$ & 11 & $1.0 \%$ \\
\hline $\begin{array}{l}\text { Zapotlán de } \\
\text { Juárez }\end{array}$ & Zapotlán de Juárez & 5 & $0.47 \%$ \\
\hline Zempoala & Zempoala & 15 & $1.4 \%$ \\
\hline \multirow[t]{2}{*}{ Otro } & Otro & 110 & $10.5 \%$ \\
\hline & TOTAL & 1,043 & $100 \%$ \\
\hline
\end{tabular}

Tabla 2: Cantidad de viajes hacia CC-UAEH por cuadrante Fuente: Elaboración propia

\section{Diseño de la encuesta}

Dadas las características de la ciudad, los objetivos que se persiguen, así como el entorno de movilidad en la universidad, la encuesta se diseña de origen a partir de 4 módulos: Módulo I: Datos socioeconómicos, Módulo II: Tendencias del uso en bici en Ciudad del Conocimiento, Módulo III: Identificación de Viajes, Módulo IV: Movilidad en la bicicleta.

\subsection{Tamaño de la muestra}

Dado que el enfoque de la investigación se presenta desde la perspectiva de los desplazamientos hacia el equipamiento de nivel superior, se optó por encuestar a la comunidad universitaria mediante correo electrónico institucional. A pesar de ser un medio digital, la efectividad de la herramienta se puede esperar que sea positiva, debido a la gran penetración de las herramientas digitales en la vida universitaria.

Así, tomando en consideración una población objetivo de 7,500 usuarios, se determina el tamaño de la muestra a partir de la siguiente ecuación (1):

$$
(1)\left\{z^{2}[p(1-p)] / e^{2}\right\} /\left\{1+\left[\left\{z^{2}[p(1-p)]\right\} / e^{2} N\right]\right\}
$$

Donde:

$$
\begin{aligned}
& M=\text { Tamaño de la muestra } \\
& N=\text { Tamaño de la población }=7500 \\
& e=\text { Margen de error }=3 \%
\end{aligned}
$$

Nivel de confianza $=95 \%$

Desviación estándar $=\mathrm{z}=1.96$

$M=935$ encuestas

De tal forma que se obtiene como resultado un total de 935 encuestas.
Para lograr el objetivo de respuesta de la encuesta sobre movilidad, se diseñó e implementó una campaña de difusión digital hacia la población objetivo, con la finalidad de enterar sobre los motivos de la encuesta (cita manual de ciclociudades) y obtener una respuesta aproximada a las tendencias del uso de la bicicleta. Los medios escogidos fueron el correo electrónico institucional, la página de facebook oficial del Instituto de Ciencias Básicas e Ingeniería (ICBI), así como la socialización mediante la comunidad de profesores mediante mensajes de telefonía celular.

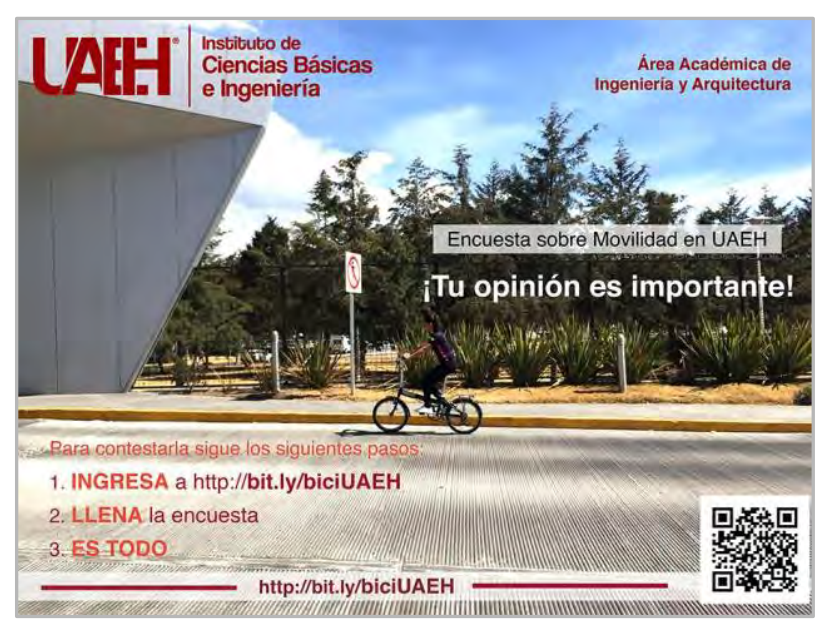

Figura 3: Cartel de difusión de la encuesta de movilidad. Fuente: Elaboración propia

\subsection{Pilotaje y aplicación de la encuesta}

Dada las características de la población objetivo, su ubicación y pertenencia a la comunidad universitaria, se optó por difundir la encuesta vía correo electrónico institucional a los miembros de la comunidad universitaria, específicamente a los que conforman el Instituto de Ciencias Básicas e Ingeniería. La principal razón radica en que dicho instituto aporta la mayoría de estudiantes, profesores, administrativos y personal de servicio que desarrollan sus actividades en la $\mathrm{CC}$ UAEH.

Se decidió que la encuesta fuera diseñada y estructurada bajo la plataforma de Google Forms, dadas las ventajas que la herramienta digital ofrece, la facilidad en la distribución de la misma, así como la utilización del correo electrónico de la comunidad universitaria de la UAEH, bajo los servicios de Google Inc.

De esta forma, en el periodo del 12 al 14 de febrero de 2018 se aplicó una primera encuesta piloto, dirigida solo a un grupo específico de estudiantes del cuarto y quinto semestre de la Licenciatura en arquitectura. El objetivo se centró en conocer las dificultades y áreas de oportunidad que presentaba el instrumento.

\subsection{Campaña de difusión de encuesta}




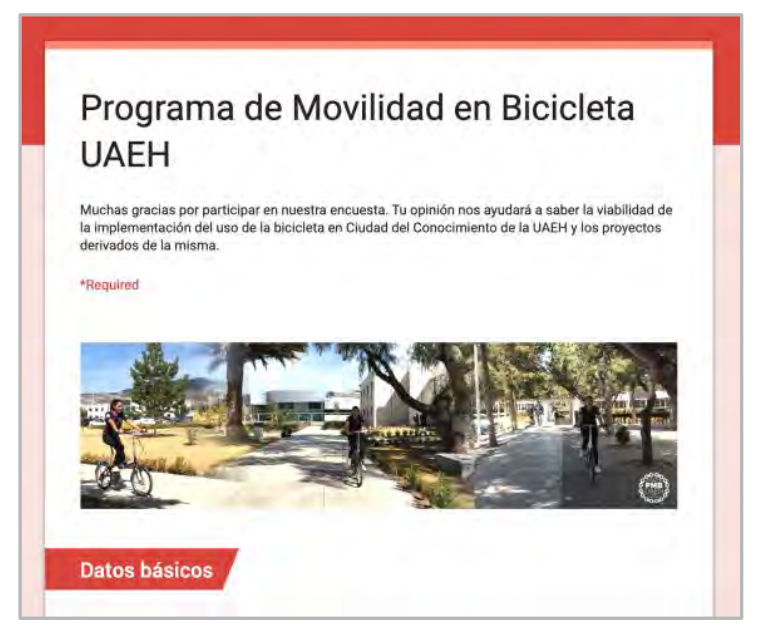

Figura 4: Portada de la encuesta online. Fuente: Elaboración propia

Finalmente, en la semana del 19 al 23 de febrero de 2018, con el apoyo de la Oficina de Comunicación Social del ICBI, mediante cuentas de correo electrónico institucionales, así como las redes oficiales del instituto, se lanzó a la comunidad la Encuesta sobre Movilidad en Ciudad del Conocimiento.

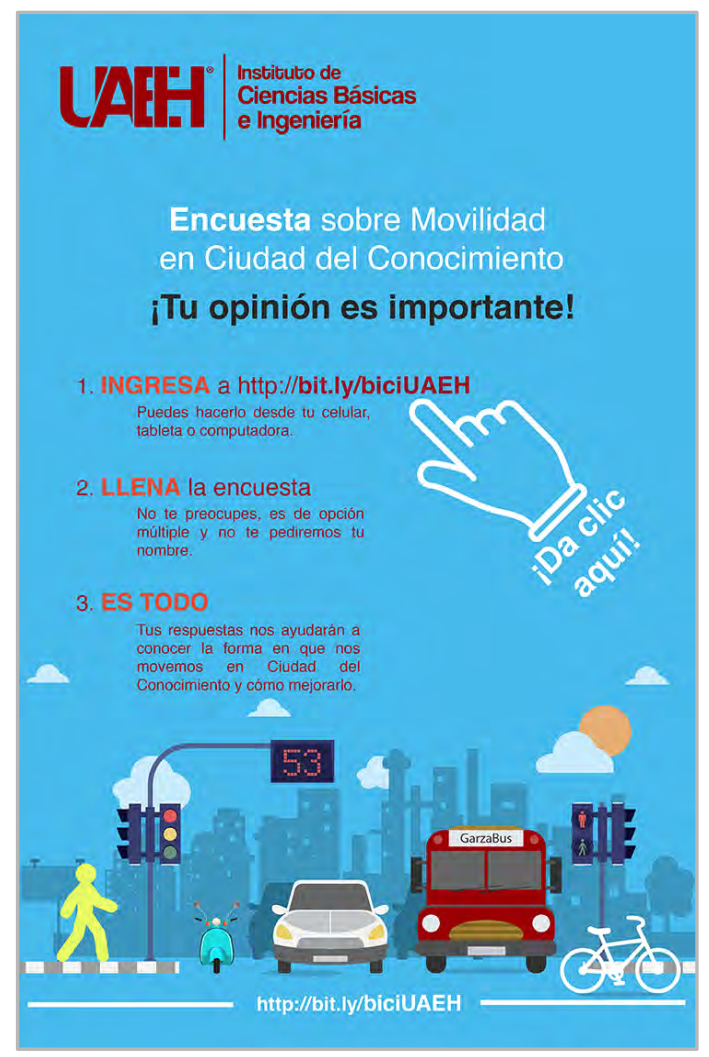

Figura 5: Flyer de difusión de la encuesta online.

Fuente: Elaboración propia

\section{Análisis de datos}

\subsection{Perfil del usuario}

De acuerdo a la encuesta digital planteada en la población estudiantil, laboral y administrativa de Ciudad del Conocimiento, UAEH, se determinó un total de $34.2 \%$ de mujeres y el $65.8 \%$ de hombres, resultando un total del $100 \%$, entre 15 y más de 60 años. Los resultados indican que el rango de edad entre 19 y 25 años es el rango de edad principal dentro de Ciudad del Conocimiento. Además, el 79.5\% corresponde a la población estudiantil a nivel licenciatura y 93.7\% pertenece al Instituto de Ciencias Básicas e Ingeniería.

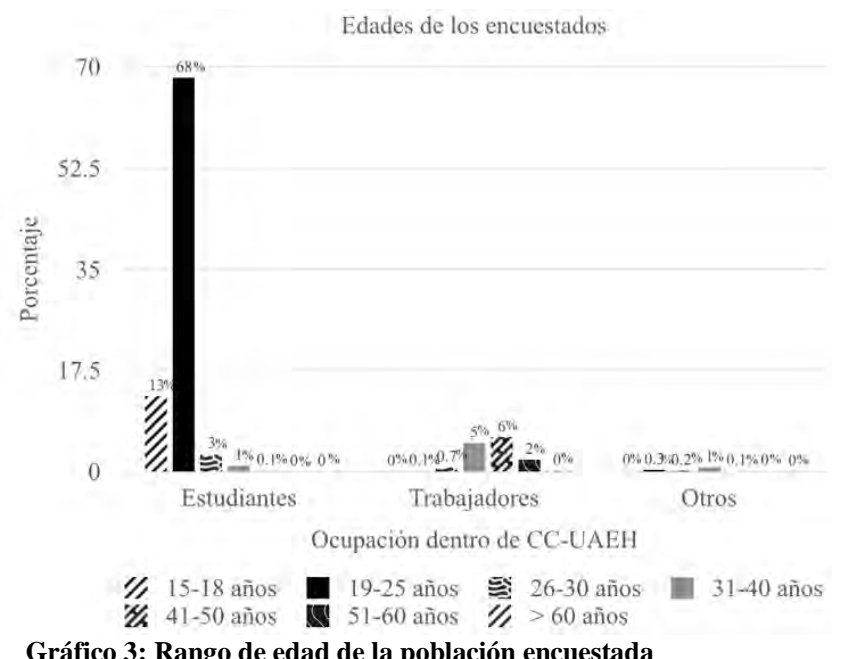

Gráfico 3: Rango de edad de la población encuestada

Fuente: Elaboración propia

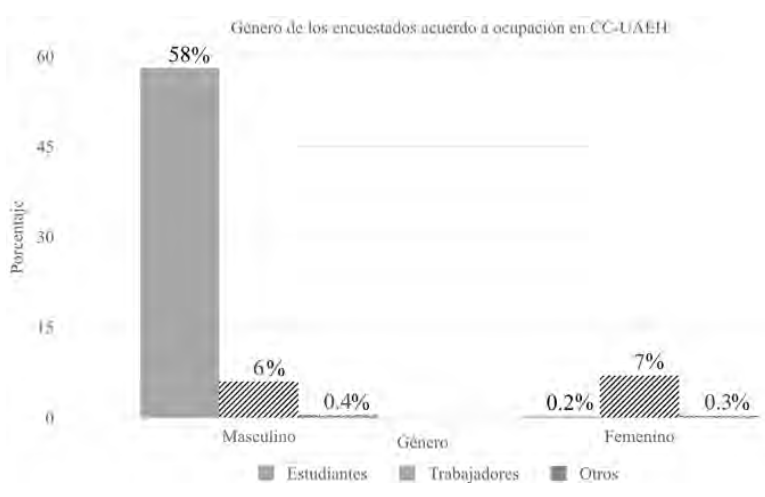

Gráfico 4: Género y ocupación de los usuarios en CC-UAEH Fuente: Elaboración propia

\subsection{Posesión de una bicicleta}

Un factor o barrera importante para la movilidad en bicicleta a tomar en cuenta es la posesión de una bicicleta, donde esta sería una barrera tangible para la población universitaria. De acuerdo a los resultados obtenidos, un $70.1 \%$ de la población estudiantil no dispone de una bicicleta, pero un $68.4 \%$ utiliza un smartphone para conectarse a internet, pero ¿qué pasaría si estas dos herramientas se combinaran? Se podrían crear rutas y recorridos en conjunto para mayor seguridad de los ciclistas, el desarrollo de aplicaciones para smartphone donde se pueda visualizar y llevar un control del uso de la bicicleta, entre otras posibilidades y estrategias para este modo de transporte; se generaría una visión sustentable de la puesta en marcha de algún proyecto para la movilidad urbana en bici.

\subsection{Tendencias del uso en bici en Ciudad del Conocimiento}

Con un $71.7 \%$ de población muestral que no conoce un sistema de bicicletas públicas, se puede desarrollar un proyecto 
de éxito dentro de Ciudad del Conocimiento, esto de acuerdo a estadísticas muestrales. Si la institución universitaria contara con un sistema de préstamo de bicicletas dentro de Ciudad del Conocimiento, un $80 \%$ de población universitaria haría uso de este por razones de estudio-trabajo e incluso, gestiones personales; todo esto con una frecuencia de 2 o más veces por semana. Como resultado, un $80 \%$ respondió que realizarían viajes internos en Ciudad del Conocimiento con una frecuencia de varias veces por semana. Otro factor importante a considerar es que un $17.2 \%$ está dispuesto a pagar anualmente de \$51 a \$100 por el servicio de bicicletas públicas.
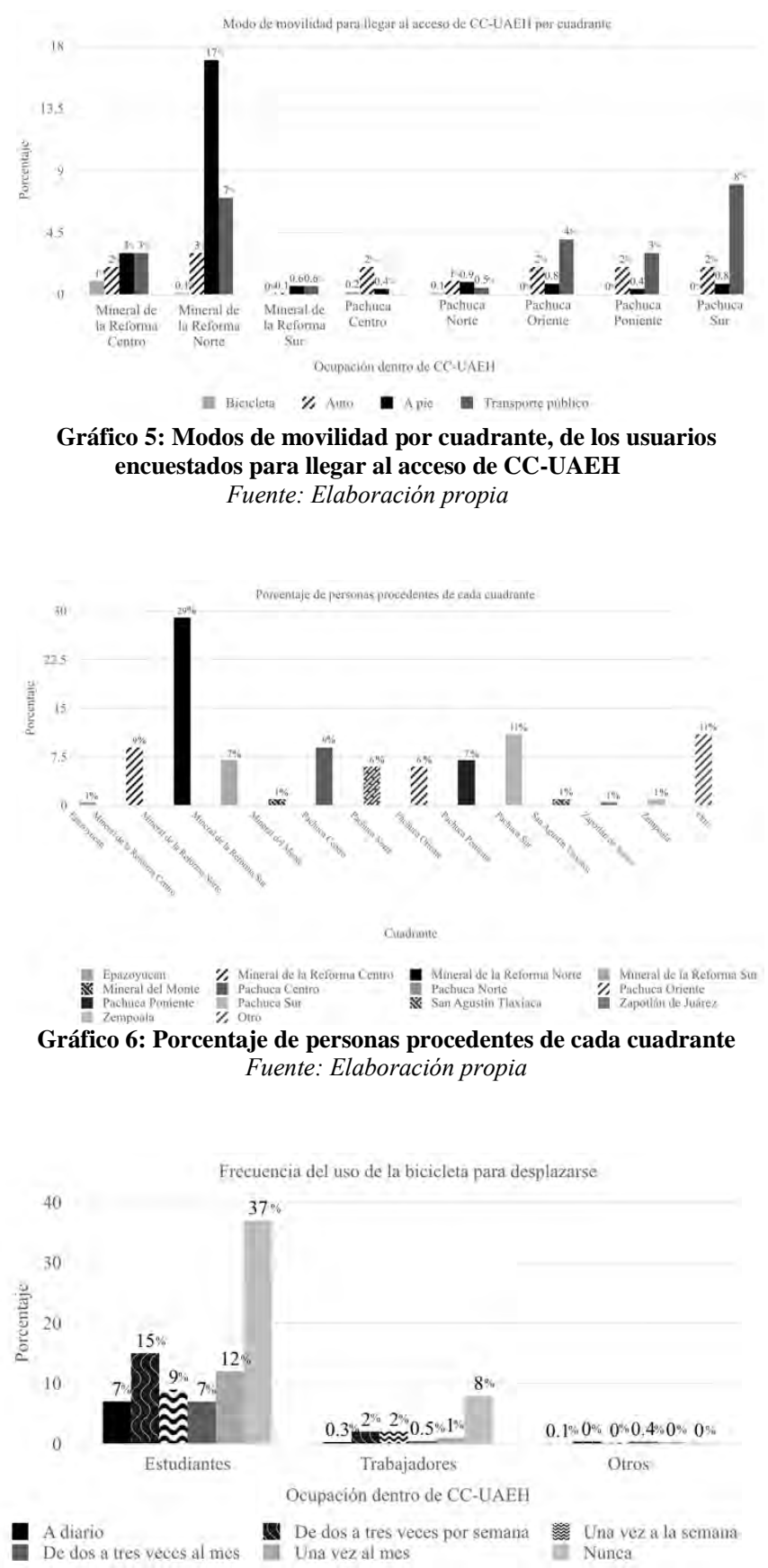

Gráfico 7: Frecuencia del uso de la bicicleta como medio de desplazamiento en los usuarios de CC-UAEH Fuente: Elaboración propia

\subsection{Identificación de Viajes}

La población universitaria llega desde diferentes puntos de partida hacia Ciudad del Conocimiento, ya sea que este sea desde casa, trabajo, gestiones de trabajo, estudio, compras, ocio, deporte o médico; en la encuesta se definieron diferentes cuadrantes en Pachuca y Mineral de la Reforma, definiéndolos como Pachuca Sur, Pachuca Norte, Pachuca Centro, Pachuca Oriente, Mineral de la Reforma Norte, Mineral de la Reforma Centro y Mineral de la Reforma Sur, dónde la mayoría de los encuestados respondió que su lugar de partida es desde su casa en un $85 \%$, además, un alto porcentaje del mismo llega desde puntos cercanos a CC-UAEH.

Es claro que el porcentaje mayor de viajes hacia $\mathrm{CC}$-UAEH se realiza desde el cuadrante de Mineral de la Reforma Norte, lo que incluye a las colonias aledañas en un radio no mayor a 3 kilómetros, que incluye a las Colonias: Carboneras, Paseo de las Reinas, Villas del Álamo, Campestre Villas del Álamo.

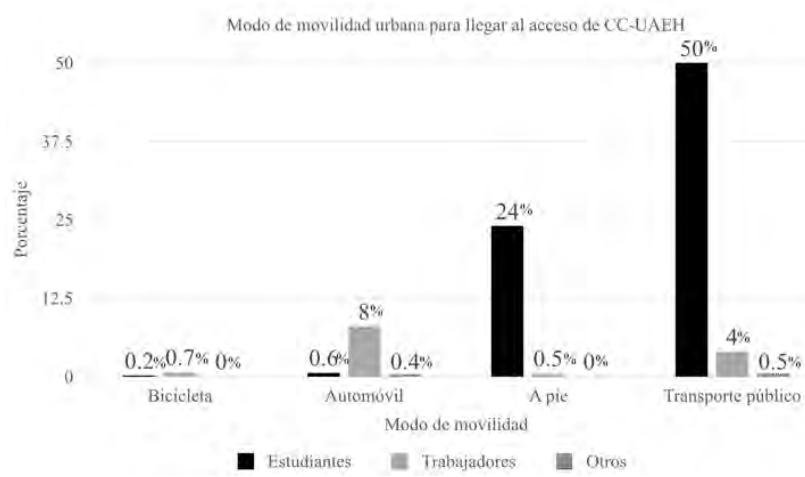

Gráfico 8: Modos de movilidad de los usuarios encuestados para llegar al acceso de CC-UAEH

Fuente: Elaboración propia

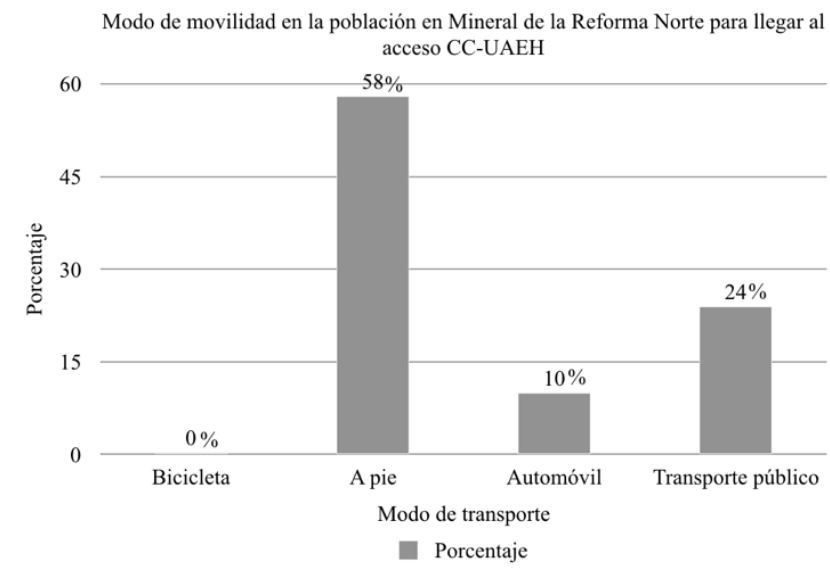

Gráfico 9: Modos de movilidad en el cuadrante Mineral de la Reforma Norte, de los usuarios encuestados para llegar al acceso de CC-UAEH Fuente: Elaboración propia

Es también interesante analizar el número de viajes realizados desde los lugares de residencia de los encuestados. En dicha muestra (gráfica 10) podemos observar que el cuadrante de Mineral de la Reforma Norte sigue siendo el lugar con mayor cantidad de viajes con éste origen. Esto puede explicarse dada la gran población estudiantil que vive en las inmediaciones de la Universidad.

Por otro lado, un $35.65 \%$ llega al acceso principal en combi y un $24.8 \%$ a pie. Mientras que un $78.6 \%$ aseguró realizar algún desplazamiento interno dentro de Ciudad del 
Conocimiento a lo largo del día, donde, el principal punto de atracción es el Pabellón Universitario con un $26.7 \%$ y en segundo lugar, con un $15.2 \%$, es la Biblioteca Central.

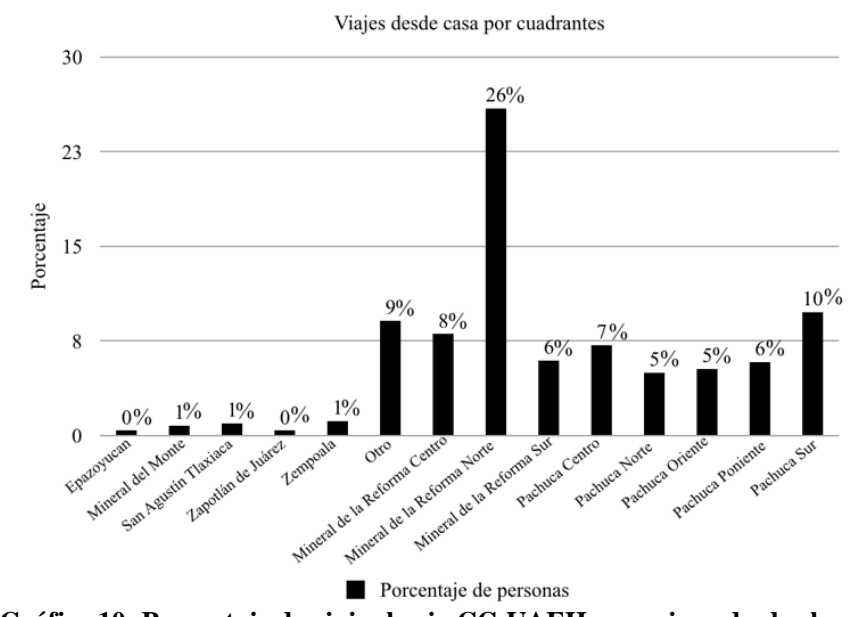

Gráfico 10: Porcentaje de viajes hacia CC-UAEH con origen desde el lugar de residencia de los usuarios

Fuente: Elaboración propia

\subsection{Potencial de uso de la bicicleta}

Tomando en cuenta el ejercicio muestral se aprecia que un que un $55.9 \%$ de la población encuestada hace uso de la bicicleta como modo de transporte para sus desplazamientos urbanos, teniendo como y el principal motivo el ejercicio o deporte con un $20.8 \%$, por ejercicio y ocio con un $22.2 \%$, y trayectorias al lugar de estudios con un $12.4 \%$. Por otra parte, un $31.9 \%$ no hace uso de la bicicleta por diferentes motivos. Pero hay razones de más peso por las cuales la población muestral revela el uso de la bicicleta que van desde la eficiencia del modo de transporte hasta impactos en la salud del usuario.

Se puede inferir de que, a pesar de que actualmente los desplazamientos en bicicleta hacia CC-UAEH son en un porcentaje menor, el uso de la bicicleta por esos mismos usuarios, en otras condiciones, se realiza.

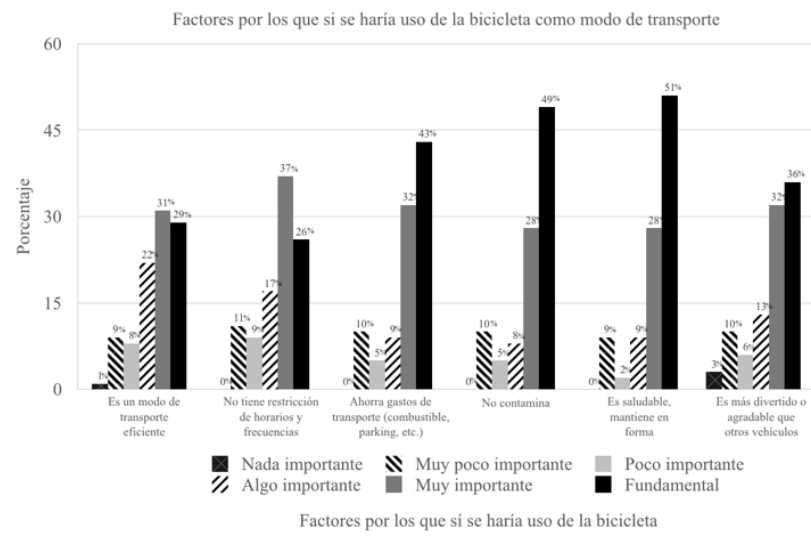

Gráfico 11: Factores por los que un usuario potencial haría uso de la bicicleta en los viajes hacia CC-UAEH Fuente: Elaboración propia

\section{Conclusiones y recomendaciones}

Con los datos analizados podemos observar que existe una gran cantidad de desplazamientos a pie hacia Ciudad del Conocimiento, pero de igual forma, la encuesta revela una gran disposición a su uso, incluso aunque los usuarios manifestaron no contar con una bicicleta. Por otra parte, las principales preocupaciones de los encuestados eran la percepción de seguridad o falta de infraestructura peatonal y ciclista.

Creemos que este ejercicio resulta en un modelo replicable para otras instituciones de educación superior (IES) debido a la practicidad de la herramienta digital, a su fácil manejo, y a las condiciones de penetración y masificación de los servicios de internet. En este sentido, es recomendable que cada institución superior establezca sus alcances particulares, ya que cada lugar presenta factores y condiciones distintas. Por otra parte, se debe tener muy claro el alcance del instrumento a implementar y se recomienda que cada instituto adapte las variables de acuerdo a las condiciones de lugar, topografía, clima, etc.

Para la UAEH se percibe una clara disposición al uso de la bicicleta como modo de transporte y es posible explorar la idea de un sistema de prestamo de bicicletas para la movilidad al interior de la institución, dado que en este tipo de equipamiento la movilidad no se limita a la puerta de acceso, si no que continua al interior del campus. Por otra parte, se contemplan múltiples beneficios al hacer uso de la bicicleta como modos de transporte, entre ellos, de salud, personales, económicos, sociales y ambientales.

El uso y fomento de la bicicleta como modo de transporte al interior de las universidades podría llegar a considerarse como una estrategia integral de salud. Además, siempre se deben de tener en mente los objetivos de las universidades y la búsqueda de los mismos.

De igual forma, dada la ubicación de la CC-UAEH con respecto a la Zona Metropolitana de Pachuca, observamos que se encuentra en los límites territoriales del Municipio de Mineral de la Reforma conturbándose con Pachuca (Cuadrante Mineral de la Reforma Norte), el municipio con mayor atracción de actividades. Esta situación la hace interesante, ya que la distancia que existe entre la ultima ciclovia del municipio de Pachuca (ubicada en el limite del Boulevard Colosio) y el acceso a la CC-UAEH es tan solo de $1.6 \mathrm{~km}$, sin embargo, este trayecto no presenta infraestructura ciclista debido a que el municipio de Mineral de la Reforma carece de una secretaria o de programas enfocados a la movilidad en bicicleta como medio de transporte. Se detecta entonces una gran posibilidad del impulso a la movilidad en bicicleta y a la movilidad metropolitana, si se atiende este tramo de conexión, lo que representaría el primer ejercicio de movilidad urbana no motorizada a nivel metropolitano.

\section{Agradecimientos}

Este trabajo ha sido realizado con el apoyo de la Dirección del Instituto de Ciencias Básicas e Ingeniería y su Oficina de Comunicación Social, así como a las distintas áreas académicas del Instituto. De igual forma, deseamos agradecer al Área Académica de Ingeniería y Arquitectura, y a la Licenciatura en Arquitectura por el apoyo en la difusión y aplicación de la encuesta. 


\section{Referencias}

Cal y Mayor \& Asociados. (2012). Proyectos y estudios del tren suburbano en la región sur del Estado de Hidalgo y zonas metropolitanas del Estado de México y Ciudad de México. Secretaria de Obras Públicas y Ordenamiento Territorial del Gobierno del Estado de Hidalgo.

Campbell, T. (2013). Beyond Smart Cities: How Cities Network, Learn and Innovate. Routledge.

Cárdenas, D. A. (2015). ¿Pachuca ciudad con movilidad sostenible? Recuperado el 16 de junio de 2016, a partir de http://www.milenio.com/firmas/david_aaron_cardenas/Pa chuca-ciudad-movilidad-sostenible $1 \overline{8} \quad 598920135 . \mathrm{html}$

Castells, M., \& Hall, P. (1994). Technopoles of the world: The making of twenty-first-century industrial complexes. Downey, J., \& McGuigan, J. (1999). Technocities: The Culture and Political Economy of the Digital Revolution. SAGE. London; New York: Routledge.

Equipo UNIBICI. (2008). Informe Proyecto UNIBICI. Universidad Politécnica de Madrid. Madrid.

Grant, J. (2006). Planning the Good Community: New Urbanism in Theory and Practice. Taylor \& Francis.

Herce, M. (2009). Sobre la movilidad en la ciudad: Propuestas para recuperar un derecho ciudadano. Reverte.

Imaz, M., Ayala, D. E., Gutiérrez, L., \& González, M. (2015). ECOPUMA, the Strategy for a Sustainable University at UNAM. En W. Leal Filho (Ed.), Transformative Approaches to Sustainable Development at Universities: Working Across Disciplines (pp. 503-511). https://doi.org/10.1007/978-3-319-08837-2_33

Ishida, T., \& Isbister, K. (Eds.). (2000). Digital cities: Technologies, experiences, and future perspectives. Berlin; New York: Springer.

Lange, C. (2011). Dimensiones culturales de la movilidad urbana. Revista INVI, 26(71), 87-106. https://doi.org/10.4067/S0718-83582011000100004
Lezama, J., \& Domínguez, J. (2006). Medio ambiente y sustentabilidad urbana. Papeles de población, 12(49), 153 176. Recuperado en 15 de octubre de 2019, de http://www.scielo.org.mx/scielo.php?script=sci_arttext\&p $\mathrm{id}=\mathrm{S} 1405-74252006000300007 \& \operatorname{lng}=\mathrm{es} \& \operatorname{tng}=\mathrm{es}$

Lizárraga, C. (2006). Movilidad urbana sostenible: Un reto para las ciudades del siglo XXI. Revista Economía Sociedad y Territorio, VI(22), 238-321.

Monzón, A., La Paix, L., \& Rondinella, G. (2008). Potencial de uso de la bicicleta en la Ciudad Universitaria de Madrid. II CIMO: "Hacia una nueva cultura de la movilidad urbana". Presentado en II CIMO - Congreso Internacional de Movilidad de Ciudadanos de Madrid., Madrid. Recuperado de http://www.fundacionmovilidad.es:8080/_archivos/_uploa d/_archivos/Monzon.pdf

Mossberger, K., Tolbert, C. J., \& Franko, W. (2013). Digital Cities: The Internet and the Geography of Opportunity. OUP USA.

Mumford, E. P. (2002). The CIAM Discourse on Urbanism, 1928-1960. MIT Press.

Telefónica, F. (2011). Smart Cities: Un primer paso hacia la internet de las cosas. Fundación Telefónica.

Townsend, A. M. (2013). Smart Cities: Big Data, Civic Hackers, and the Quest for a New Utopia. W. W. Norton \& Company.

Vilela, M., Grimoni, J., \& Burani, G. (2010). Sustainable Campus Program: University of Sao Paulo and its contribution to the reduction of environmental and economic impacts. Recuperado de https://www.osti.gov/etdeweb/biblio/22028656

UAEH (2018). Infraestructura Universitaria. Recuperado el 4 de octubre de 2019, de Universidad Autónoma del Estado de Hidalgo website: http://sgc.uaeh.edu.mx/proyectosobras/index.php/infraestructura-universitaria 